\title{
Role of acute kidney injury biomarkers to guide renal replacement therapy initiation, what we learn from EARLY-RRT trial and FST trial?
}

\author{
Nattachai Srisawat ${ }^{1}$, Kamonwan Tangvoraphonkchai ${ }^{2}$, Nuttha Lumlertgul ${ }^{1}$, Kriang Tungsanga $^{1}$, \\ Somchai Eiam-Ong ${ }^{1}$
}

${ }^{1}$ Division of Nephrology, Department of Medicine, Faculty of Medicine, Chulalongkorn University, King Chulalongkorn Memorial Hospital, Bangkok, Thailand; ${ }^{2}$ Department of Medicine, Faculty of Medicine, Mahasarakham University, Mahasarakham, Thailand

Correspondence to: Nattachai Srisawat, MD, MSc. Division of Nephrology, Department of Medicine, Faculty of Medicine, Chulalongkorn University, King Chulalongkorn Memorial Hospital, Bangkok, Thailand. Email: drnattachai@yahoo.com.

Provenance: This is an invited article commissioned by the Section Editor Xue-Zhong Xing [National Cancer Center (NCC)/Cancer Hospital, Chinese Academy of Medical Sciences (CAMS) and Peking Union Medical College (PUMC), Beijing, China].

Response to: Hoste EA, Vandenberghe W. Plasma neutrophil gelatinase-associated lipocalin (NGAL) for timing of initiation of renal replacement therapy for acute kidney injury? J Thorac Dis 2018;10:S3989-93

Meersch M, Zarbock A, Küllmar M. Renal biomarkers for the initiation of renal replacement therapy-is this the future? J Thorac Dis 2018;10:S3229-32.

Submitted Nov 07, 2018. Accepted for publication Nov 16, 2018.

doi: $10.21037 /$ jtd.2018.11.95

View this article at: http://dx.doi.org/10.21037/jtd.2018.11.95

We wrote this letter to the editor in response to the commentary by Hoste et al., and Meersch et al., on our trial "The effect of early Renal Replacement Therapy guided by plasma Neutrophil Gelatinase Associated Lipocalin on outcome of Acute Kidney Injury: a feasibility study (EARLY-RRT)" trial which was recently published in the $\mathcal{f}$ Crit Care (1). In brief, we divided the study into two phases, triage and interventional phase, running subsequently. As a guide for triage to renal replacement therapy (RRT), we measured plasma neutrophil gelatinase associated lipocalin (pNGAL) at the enrollment time. Forty patients with pNGAL $\geq 400 \mathrm{ng} / \mathrm{mL}$ (high pNGAL group) were randomized to 'early' or 'standard' group. Patients with pNGAL $<400 \mathrm{ng} / \mathrm{mL}(\mathrm{n}=20)$ were defined as low pNGAL group. The triggering pNGAL selected acute kidney injury (AKI) patients with more severity of illness and worse clinical outcome. However, in high pNGAL group, early RRT did not result in different 28-day mortality from the standard group. The median numbers of day free from mechanical ventilation were significantly higher in the early RRT group.

We delighted to learn that Hoste et al., and Meersch et al. do agree that the strongest point in our study is the concept of the study and the study design. Although dialysis was established over six decades, the issue of early versus standard RRT in AKI remains unsolved. Indeed, nearly sixty studies have been conducted on this issue the majority of these studies are observational design, which favored early RRT. However, these studies might have selection bias as some AKI patients who did not receive RRT due to either self-recovery or death, would not be recruited. Moreover, some residual confounders, inhomogeneous baseline, and diversity in general and RRT practice, which are usually neglected in observational study, may affect the results rather than the intervention per se. In 2017, there were 6 meta-analyses (2-8) and 3 major randomized controlled trials (AKIKI, ELAIN, IDEAL-ICU) (9-11) which aimed to test the benefit of early RRT and showed the conflicting results. Of note, most of the previous studies did not use the risk stratification strategy to identify the high-risk AKI patients with poor renal self-recovery. Because RRT is not the benign procedure. We strongly do believe that screening the high-risk patients will help us to exclude low risk AKI patients from unnecessary RRT exposure. Therefore, we have conducted the first feasibility study to explore the 
optimal timing of RRT initiation guided by a novel AKI biomarker.

To response for the concern from Meersch et al. regarding the severity of our patients which is quite low. In fact, in the interventional trial (high pNGAL group), the mean values of APACHE II score and SOFA score, and pNGAL levels were 18,9 , and $972 \mathrm{ng} / \mathrm{mL}$ which are in the moderate severity. Regarding to the concerning of the efficacy of CRRT in our study, the mean duration of CRRT in our study was 16 hours. In this regard, Uchino et al., reported that on average at least $16 \mathrm{~h}$ per day of CRRT was required to maintain creatinine and urea concentration for each 24-h cycle (12). Therefore, CRRT without anticoagulant in our study was still effective.

We chose pNGAL for many reasons. Firstly, it is one of most robust AKI biomarkers which was tested and validated. Secondly, we can use pNGAL as a point of care testing. Thirdly, we reported the capability of pNGAL in predicting renal recovery in a cohort of severe AKI from pneumonia (13). Currently, we do not have the standard cutoff point for pNGAL in predicting renal recovery. We selected the pNGAL cutoff point at $400 \mathrm{ng} / \mathrm{mL}$ because at this cutoff point valued show the high specificity and high positive predictive value (14). We agree with the comment from Meersch et al. that high cutoff point ( $>600 \mathrm{ng} / \mathrm{mL}$ ) might show the higher specificity. However, if we chose the higher cutoff point, this mean that the timing of early RRT will be delayed, too. In our study, we clearly demonstrated that all AKI patients in the low pNGAL group (pNGAL $<400 \mathrm{ng} / \mathrm{mL}$ ) did not require any RRT. This suggest that pNGAL is a reliable marker to triage AKI patients. However, we agree with the comment from Hoste et al. and Meersch et al. that pNGAL is not the perfect marker and we still need to find out the more reliable biomarkers.

Recently, we published the study "early versus standard initiation of renal replacement therapy in furosemide stress test non-responsive acute kidney injury patients (the FST trial)" (14). We used the furosemide stress test (FST) to identify the high risk for AKI progression. FST was performed followed the previous study by Chawla et al. by injection $1 \mathrm{mg} / \mathrm{kg}$ of furosemide in furosemidenaive patients (or $1.5 \mathrm{mg} / \mathrm{kg}$ in previous furosemide users) in patients who were admitted in intensive care unit (ICU) and were diagnosed as AKI by the KDIGO criteria (15).
The outcomes were compared between patients who passed urine $<200 \mathrm{~mL}$ (FST-nonresponsive) and $\geq 200 \mathrm{~mL}$ (FST-responsive) in 2 hours. The survival rate was significantly higher among FST-responsive patients. Then, FST-nonresponsive patients were randomized to start RRT within 6 hours (early group) or when any conventional indication for RRT was met (standard group). Again, there were no differences in mortality and dialysis dependence at 28 days, renal recovery, and ICU-free day between early and standard RRT. Of note, only 6/44 (13.6\%) of FSTresponsive patients subsequently underwent RRT. We were able to select a group with a $45 / 60(75 \%)$ RRT rate in the standard RRT group of FST nonresponders. It is tempting to speculate that FST can be used to stratify patients who have low risk for AKI progression and RRT requirement.

After the results of the aforementioned major RCTs, it seems likely that early RRT strategy is still need more evident to support and "wait and see" strategy should be the optimal approach to use for current practice. However, as mentioned by AKIKI study team, active monitoring high risk AKI patients is still very crucial. Data from AKIKI trial, FST trial and IDEAL-ICU trial showed that the highest mortality $(61.8 \%, 64.4 \%$, and $68 \%$, respectively) was found among patients in the "wait and see" (standard) group who received RRT. However, this finding may reflect a subgroup of patients with more severe underlying disease. We cannot conclude that death was related to the delay in RRT or that earlier initiation of RRT would provide a survival benefit. But we strongly believe that we need some tools/markers to monitor the high-risk patients for AKI progression if we will use "wait and see" approach. Based on our finding from EARLY-RRT trial and FST trial, we proposed to use pNGAL and FST as one of the markers to use for active surveillance high risk AKI patients.

In summary, the issue of early and standard RRT is still debatable as there are no consensus criteria, indicator or time point to start RRT. Although our studies could not define the optimal point for RRT initiation, we have proposed two interventions to designate safety margin for the "wait and see" strategy. Both pNGAL $<400 \mathrm{ng} / \mathrm{mL}$ and FST-responsiveness can indicate a good prognosis of AKI which the need for RRT is less likely. However, these tests need to be validated with larger sample size before clinical application (Figure 1). 

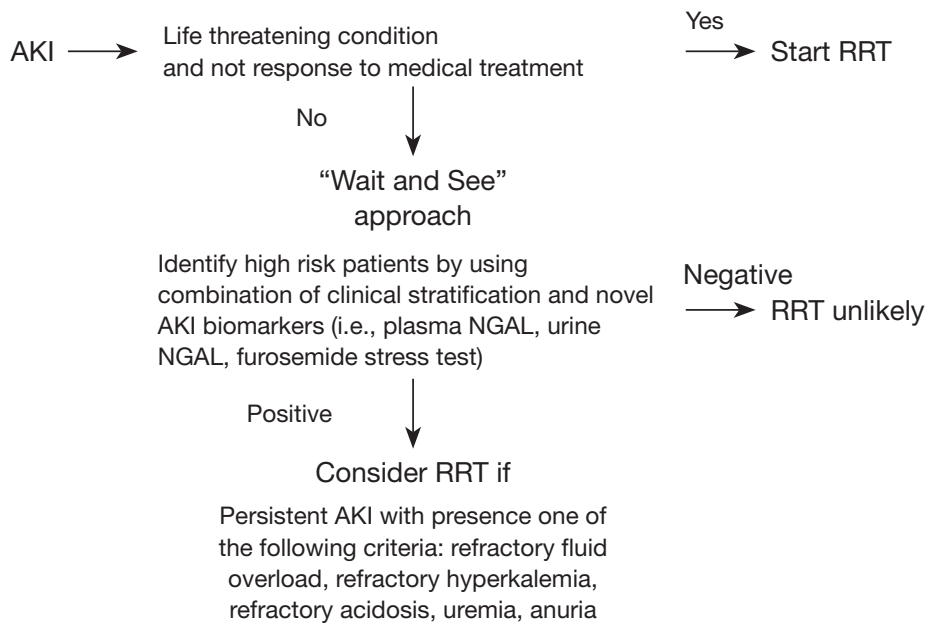

Figure 1 Proposed algorithm for renal replacement therapy initiation in AKI patients. AKI, acute kidney injury; RRT, renal replacement therapy; pNGAL, plasma neutrophil gelatinase-associated lipocalin; FST, furosemide stress test.

\section{Acknowledgements}

None.

\section{Footnote}

Conflicts of Interest: The authors have no conflicts of interest to declare.

\section{References}

1. Srisawat N, Laoveeravat $\mathrm{P}$, Limphunudom $\mathrm{P}$, et al. The effect of early renal replacement therapy guided by plasma neutrophil gelatinase associated lipocalin on outcome of acute kidney injury: A feasibility study. J Crit Care 2018;43:36-41.

2. Yang XM, Tu GW, Zheng JL, et al. A comparison of early versus late initiation of renal replacement therapy for acute kidney injury in critically ill patients: an updated systematic review and meta-analysis of randomized controlled trials. BMC Nephrol 2017;18:264.

3. Lai TS, Shiao CC, Wang JJ, et al. Earlier versus later initiation of renal replacement therapy among critically ill patients with acute kidney injury: a systematic review and meta-analysis of randomized controlled trials. Ann Intensive Care 2017;7:38.

4. Bhatt GC, Das RR. Early versus late initiation of renal replacement therapy in patients with acute kidney injury-a systematic review \& meta-analysis of randomized controlled trials. BMC Nephrol 2017;18:78.
5. Wang C, Lv LS, Huang H, et al. Initiation time of renal replacement therapy on patients with acute kidney injury: A systematic review and meta-analysis of 8179 participants. Nephrology (Carlton) 2017;22:7-18.

6. Feng YM, Yang Y, Han XL, et al. The effect of early versus late initiation of renal replacement therapy in patients with acute kidney injury: A meta-analysis with trial sequential analysis of randomized controlled trials. PLoS One 2017;12:e0174158.

7. Bagshaw SM, Wald R. Strategies for the optimal timing to start renal replacement therapy in critically ill patients with acute kidney injury. Kidney Int 2017;91:1022-32.

8. Yang XM, Tu GW, Zheng JL, et al. A comparison of early versus late initiation of renal replacement therapy for acute kidney injury in critically ill patients: an updated systematic review and meta-analysis of randomized controlled trials. BMC Nephrol 2017;18:264.

9. Gaudry S, Hajage D, Schortgen F, et al. Initiation Strategies for Renal-Replacement Therapy in the Intensive Care Unit. N Engl J Med 2016;375:122-33.

10. Zarbock A, Kellum JA, Schmidt C, et al. Effect of Early vs Delayed Initiation of Renal Replacement Therapy on Mortality in Critically Ill Patients With Acute Kidney Injury: The ELAIN Randomized Clinical Trial. JAMA 2016;315:2190-9.

11. Barbar SD, Clere-Jehl R, Bourredjem A, et al. Timing of Renal-Replacement Therapy in Patients with Acute Kidney Injury and Sepsis. N Engl J Med 2018;379:1431-42.

12. Uchino S, Fealy N, Baldwin I, et al. Continuous is not continuous: the incidence and impact of circuit "down- 
time" on uraemic control during continuous veno-venous haemofiltration. Intensive Care Med 2003;29:575-8.

13. Srisawat N, Murugan R, Lee M, et al. Plasma neutrophil gelatinase-associated lipocalin predicts recovery from acute kidney injury following community-acquired pneumonia. Kidney Int 2011;80:545-52.

14. Lumlertgul N, Peerapornratana S, Trakarnvanich T, et al. Early versus standard initiation of renal replacement therapy in furosemide stress test non-responsive acute kidney injury patients (the FST trial). Crit Care 2018;22:101.

15. Chawla LS, Davison DL, Brasha-Mitchell E, et al. Development and standardization of a furosemide stress test to predict the severity of acute kidney injury. Crit Care 2013;17:R207.

Cite this article as: Srisawat N, Tangvoraphonkchai K, Lumlertgul N, Tungsanga K, Eiam-Ong S. Role of acute kidney injury biomarkers to guide renal replacement therapy initiation, what we learn from EARLY-RRT trial and FST trial? J Thorac Dis 2018;10(12):E835-E838. doi: 10.21037/jtd.2018.11.95 